\title{
Über den Lipasegehalt des Harns und des Blutserums bei Nierentuberkulose, mit besonderer Berück= sichtigung des separierten Harns.
}

\author{
Von \\ Rikuro Yasuda. \\ (安田陸郎) \\ (Aus der Chirurgischen Klinik von Prof. Sh. Sugimura \\ an der Universität, Sendai.)
}

Über die Serumlipase bei Tuberkulose gibt es schon Veröffentlichungen von Internisten wie auch von Chirurgen. Autoren wie Frisch u. Kollert, Tsuji, Rosowsky, Bantin, Berg, Nicolau u. Antinescu u. a. untersuchten die Serumlipase bei Lungentuberkulose. Frisch u. Kollert fanden, dass hierbei die Schwankungen des Serumlipasetiters mit dem allgemeinen Zustand des Kranken beinah parallel gehen, was dann auch von anderen Autoren bestätigt wurde. Schranz, Sorkin, Molcanovau. Nizovskaja stellten Untersuchungen über Serumlipase bei Knochen- und Gelenktuberkulose, Irger u. Sapiro bei verschiedenen chirurgischen Krankheiten und Sueoka bei chirurgischer Tuberkulose an. Schranz untersuchte die Serumlipase vor und nach Entfernung der isolierten, tuberkulösen Knochenherde und kam zu dem Schluss, dass der Lipasetiter mit der Besserung des Allgemeinbefindens sowie des lokalen Herdes durch operatives Vorgehen parallel ansteigt, im entgegengesetzten Falle dagegen sinkt. Takeba y a shi stellte höheren Lipasegehalt bei tuberkulösem Granulationsgewebe sowie tuberkulösem Eiter als bei nicht tuberkulösem Granulationsgewebe und nicht tuberkulösem Eiter, Im ok aw a dagegen stärkere Lipasewirkung bei akut-entzündlichem Eiter fest. Über die Lipase des Harns und zwar bei Tuberkulösen liegt bisher nur sehr wenig Literatur vor.

Beim Durchmustern der Literatur bemerken wir schon, dass es Berichte über die Lipase des Serums und des Harns bei Nierentuberkulösen kaum gibt. Bei offener Nierentuberkulose werden die Krankheitsprodukte aus den tuberkulösen Herden der einen oder der anderen 
Niere mit dem Harn ausgeschieden. Wie sich hierbei die Lipase in separierten Harnen verhält, darüber liegt bischer keine Untersuchung vor. Zur Ausfüllung dieser Lücke habe ich zuerst den Lipasetiter des Harns und des Blutserums bei Gesunden, dann bei Tuberkulösen mit Ausschluss von Nierenerkrankungen und bei Nierentuberkulösen und endlich in separierten Harnen sowohl bei Nierentuberkulose als auch bei sonstigen chirurgischen Nierenerkrakungen bestimmt. Es sei hier bemerkt, dass bei meinen Kranken Komplikationen durch den Verdauungstraktus, vor allem auch das Pankreas, die Leber und die Milz ganz ausgeschlossen waren. Ausserdem wurde der Lipasegehalt auch in tuberkulösem sowie nichttuberkulösem Eiter untersucht. Die Ergebnisse seien im Folgenden kurz wiedergegeben.

\section{Technische Bemerkungen.}

Bei meinen Lipaseuntersuchungen des Harns und des Blutserums bediente ich mich der von Takebay ashi modifizierten Tributyrinmethode nach Rona u. Michaelis. Hierbei verwendete ich nach Nakajima u. Mizuno eine Bruttemperatur von $40^{\circ} \mathrm{C}$ statt $38^{\circ} \mathrm{C}$.

Der Gesamtharn wurde bei Gesunden und Kranken in einer bestimmten Vormittagsstunde und nach Fasten von mindestens $2 \frac{1}{2}$ bis 3 Stunden nach dem Frühstück, d.h. von 10 bis $10 \frac{1}{2}$ Uhr gesammelt. In der Zwischenzeit wurde das Blut den Armvenen entnommen. Den Kranken wurde ausserdem einen Tag vorher Arzeneimittelaufnahme verboten und bei Fiebernden die Untersuchung zu möglichst fieberfreier Zeit vorgenommen. Bei den separierten Harnen wurde der Ureterenkatheterismus sowohl in der Vormittags- als auch in der Nachmittagsstunde, aber möglichst nach kürzerem Fasten nach der Mahlzeit ausgeführt.

Bei Gesunden wie Kranken wurden der Gesamtharn oder die separierten Harne ausser auf Lipasetiter auch, wie üblich, auf spezifisches Gewicht, Reaktion und auf pathologische chemische und morphologische Bestandteile untersucht. Auch wurden bei sämtlichen Fällen, und zwar bei separierten Harnen, die verschiedenen üblichen Methoden der Nierenfunktionsprüfung parallel angestellt. Es sei hier bemerkt, dass bei der Lipaseuntersuchung der Harn in nativem Zustaude, das Blutserum in 10 facher Verdünnung unter Zusatz einer 0,9\% igen physiologischen Kochsalzlösung und der Eiter in 5facher Verdünnung durch physiologischer Kochsalzlösung, von jedem $2 \mathrm{ccm}$, verwendet wurde.

\section{Ergebnisse meiner Untersuchungen.}

Es standen mir 15 Gesunde vom 16. bis 51. Lebensjahr, beiderlei Geschlechts, zur Verfügung. Der Lipasetiter des Gesamtharns und des Blutserums all dieser, bei denen ein tuberkulöses Leiden klinisch ganz ausgeschlossen war, wurde bestimmt. Die Zahlen gibt Tabelle I wieder. 
Tabelle I. Lipase im Harn und Blutserum bei Gesunden.

\begin{tabular}{|c|c|c|c|}
\hline \multirow{2}{*}{ Fall-Nr. } & \multirow{2}{*}{$\begin{array}{l}\text { Name, Alter } \\
\text { n. Geschlecht }\end{array}$} & \multicolumn{2}{|c|}{ Lipase (k) in } \\
\hline & & Harn & Blutserum \\
\hline $\begin{array}{r}1 \\
2 \\
3 \\
4 \\
5 \\
6 \\
7 \\
8 \\
9 \\
10 \\
11 \\
12 \\
13 \\
14 \\
15\end{array}$ & $\begin{array}{llll}\text { M. H. } & 16 & \mathrm{~m} \\
\text { N. S. } & 18 & \mathrm{~m} \\
\text { K. S. } & 18 & \mathrm{~m} \\
\text { S. O. } & 18 & \mathrm{~m} \\
\text { T. M. } & 20 & \mathrm{~m} \\
\text { T. S. } & 20 & \mathrm{w} \\
\text { M. M. } & 22 & \mathrm{~m} \\
\text { K. T. } & 23 & \mathrm{~m} \\
\text { H. S. } & 23 & \mathrm{w} \\
\text { I. T. } & 26 & \mathrm{~m} \\
\text { J. T. } & 30 & \mathrm{~m} \\
\text { K. K. } & 37 & \mathrm{~m} \\
\text { U. T. } & 40 & \mathrm{~m} \\
\text { T. K. } & 45 & \mathrm{~m} \\
\text { T. M. } & \mathbf{5 1} & \mathrm{m}\end{array}$ & $\begin{array}{r}0,000466 \\
340 \\
1369 \\
782 \\
841 \\
782 \\
509 \\
782 \\
340 \\
500 \\
509 \\
715 \\
782 \\
841 \\
782\end{array}$ & $\begin{array}{r}0,003825 \\
3825 \\
3600 \\
3825 \\
4327 \\
4763 \\
4067 \\
4067 \\
1666 \\
4606 \\
4327 \\
3825 \\
4067 \\
3600 \\
4067\end{array}$ \\
\hline & sehnitt & $\begin{array}{c}0,0006893 \\
\mathrm{E}(\mathbf{M})= \pm 0,00004355\end{array}$ & $\begin{array}{c}0,0038612 \\
\mathrm{E}(\mathrm{M})= \pm 0,00011393\end{array}$ \\
\hline
\end{tabular}

Danach betrug die Durchschnittszahl des Lipasetiters im gesunden Harn 0,0006893, die des Blutserums bei Gesunden 0,0038612. Der Unterschied zwischen dem Maximum und dem Minimum des Lipasetiters war bei normalem Harn über 2fach grösser als der beim normalen Blutserum. Sagal bemerkte schon, was später auch Autoren wie Tsuji, Schranz, Kura mochi und Sueok a bestätigten, dass der Lipasegehalt des normalen Serums ganz konstant und unabhängig von der Nahrung ist, und dass die Lipaseaktivität mit zunehmendem Lebensalter allmählich abnimmt. Auch stellte Tsuji fest, dass der Lipasegehalt des normalen Serums vom Geschlecht, Lebensalter und von der Ernährung ganz unabhängig ist. Dass Lipase im normalen Harn fehlt oder nur in Spuren enthalten ist, wurde zuerst von T anfani (zitiert bei $O p$ p en h e imer) angegeben. Es gibt aber seitdem kaum Angaben über den Lipasegehalt des normalen Harns.

Ich bestimmte ferner den Lipasetiter des Harns und Blutserums bei 15 Kranken mit chirurgischer sowie innerer Tuberkulose ohne Nierenerkrankungen (siehe Tabelle II). Hierbei betrug die Durchschnittszahl des Lipasetiters im Harn 0,0009251 und im Blutserum 0,0040215. Man bemerkt also, dass der Lipasetiter des Harns und des Blutserums bei Tuberkulösen ohne Nierenerkrankungen durchschnittlich weit höher als bei Gesunden ist. Die Schwankungen zwischen den einzelnen Lipasetitern waren beim Harn über 2 fach grösser als beim Blutserum. 
Tabelle II. Lipase im Harn und Blutserum bei Tuberkulösen mit Ausschluss der Nierenerkrankungen.

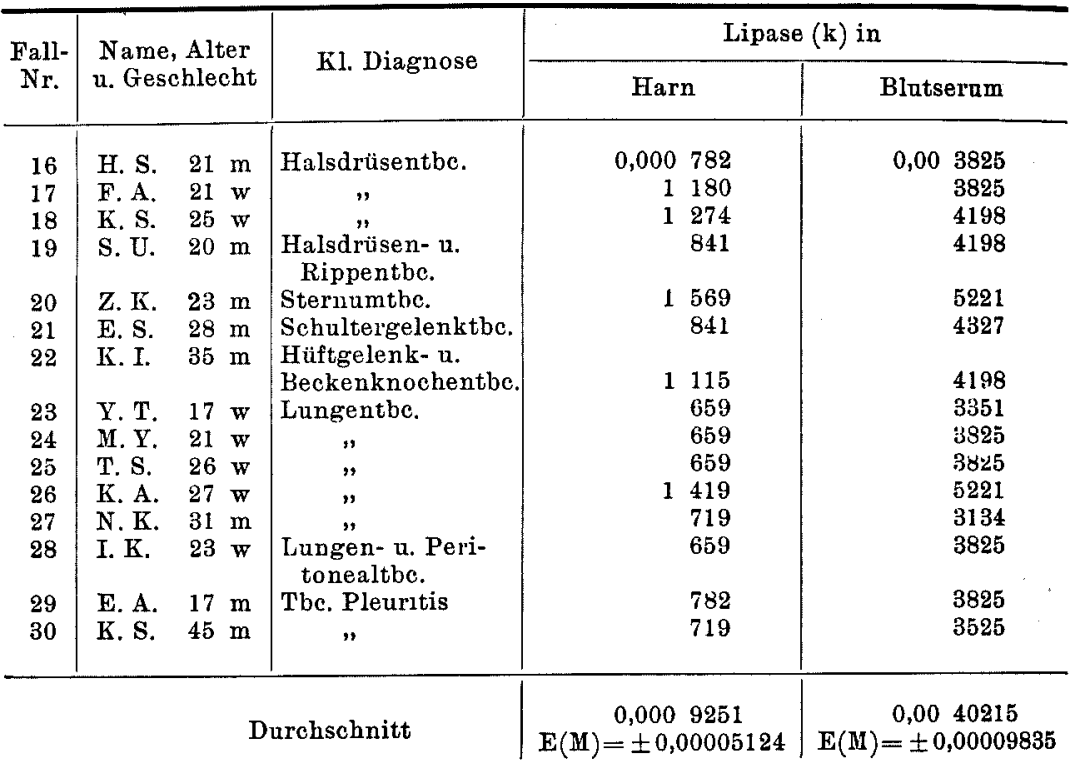

Durch Untersuchungen vieler Autoren wie Bauer, Frisch u. Kollert, Tsuji, Rosowsky, Bantin, Nicolau u. Antinescu und Berg hat sich herausgestellt, dass der Serumlipasetiter bei Tuberkulösen und zwar Lungentuberkulösen, ebenso wie bei Gesunden, individuellen Schwankungen unterworfen ist, das Ansteigen des lipolytischen Vermögens des Bluts hierbei in der Regel als ein günstiges prognostisches Zeichen zu verwerten ist, und dass, je weiter die Lungenveränderungen schon fortgeschritten, der Lipasetiter umso niedriger ist.

Was die Serumlipase bei chirurgischen Erkrankungen und zwar bei chirurgischer Tuberkurose betrifft, so bernerkte Bauer bei Karzinomkranken, wie bei Phthisikern, in der Regel auffallende Abnahme des Serumlipasegehalts und bei Lues und Based ow scher Krankheit etwas Verminderung. Molcanova u. Nizovskaja, Sorkin und $\mathrm{Ni}$ colau u. Antinescu untersuchten Serumlipase bei Knochentuberkulose. Sorkin stellte bei $64 \%$ seiner Fälle von Knochen-, Gelenkund Drüsentuberkulose subnormalen Gehalt an Serumlipase fest. Nach Nicolau u. Antinescu verhielt sich die Serumlipase bei der Knochentuberkulose fast wie bei Lungentuberkulose. Dasselbe konnte Sueoka auch bei chirurgischer Tuberkulose, allerdings mit einigen Ausnahmen, feststellen. Schranz beobachtete, dass bei chirurgischer Tuberkulose nach radikaler Entfernung der tuberkulösen Herde der Lipasetiter mit der allgemeinen Besserung und fortschreitenden Hei- 
lung anstieg. Auch Irger u. Sapiro bemerkten bei ihren chirurgischen Erkrankungen, worunter sich Fälle maligner Tumoren, eitriger Infektionen usw. fanden, dass die lipolytische Energie des Blutserums hierbei im Vergleich zur Norm vermindert ist, dass die Senkung des Lipaseindexes auch parallel mit dem Akutwerden des Krankheitsprozesses geht, dass dagegen seine Steigerung als günstiges Zeichen für Besserung anzusehen ist. In meinen Fällen von Tuberkulose wurde der Lipasetiter des Blutserums, wie erwähnt, im Vergleich zur Norm durchschnittlich etwas erhöht gefunden. Unter Ausschluss von Pankreaserkrankungen kann die Lipase des Harns nach Oppenheimer vermehrt sein, wenn die Blutlipase vermehrt ist, wie z. B. bei Fieber, Zerfall von Leukozyten, Verdauungsstörungen usw., ausserdem auch bei Polyurien. Ich habe bei meinen Tuberkulösen, unter Ausschluss von Nierenerkrankungen, durchschnittlich nur etwas erhöhte Werte des Lipasetiters des Harns festgestellt.

Ferner habe ich 10 pyogene und 14 tuberkulöse Eiter auf ihren Lipasegehalt untersucht (siehe Tabelle III u. IV). Die Durchschnitts-

Tabelle III. Lipase in nichttuberkulösem Eiter.

\begin{tabular}{|c|c|c|c|c|}
\hline $\begin{array}{l}\text { Fall- } \\
\text { Nr. }\end{array}$ & $\begin{array}{l}\text { Name, Alter } \\
\text { u. Geschlecht }\end{array}$ & Kl. Diagnose & $\begin{array}{l}\text { Lipase (kj) } \\
\text { im Eiter }\end{array}$ & $\begin{array}{l}\text { Erreger } \\
\text { im Eiter }\end{array}$ \\
\hline 31 & K. S. $22 \mathrm{~m}$ & $\begin{array}{l}\text { Perforationsperitonitis } \\
\text { bei Appendizitis }\end{array}$ & 0,005915 & Colibazillen \\
\hline 32 & H. F. $25 \mathrm{~m}$ & $"$ & 1180 & $"$ \\
\hline $\begin{array}{l}33 \\
34\end{array}$ & $\begin{array}{ll}\text { M. T. } & 35 \mathrm{~m} \\
\text { T. S. } & 13 \mathrm{~m}\end{array}$ & $\begin{array}{l}\text { Subkut. Phlegmone am } \\
\text { Unterschenkel }\end{array}$ & $\begin{array}{l}1777 \\
3028\end{array}$ & $\begin{array}{l}\text { Staphyloc. } \\
\text { pyog. aureus }\end{array}$ \\
\hline 35 & M. K. $23 \mathrm{~m}$ & $\begin{array}{l}\text { Myositis purul. acuta } \\
\text { glataei }\end{array}$ & 1777 & " \\
\hline 36 & T. H. $12 \mathrm{~m}$ & $\begin{array}{l}\text { Subkut. Abszesse am } \\
\text { Kopf }\end{array}$ & 4847 & $\begin{array}{l}\text { Streptoc. } \\
\text { haemolyticus }\end{array}$ \\
\hline 37 & Z. T. $\quad 35 \mathrm{~m}$ & $\begin{array}{l}\text { Perforationsperitonitis } \\
\text { bei Appendizitis }\end{array}$ & 5221 & $\begin{array}{l}\text { Streptoe. } \\
\text { viridans }\end{array}$ \\
\hline 38 & Y. F. $17 \mathrm{~W}$ & Metapneum. Empyem & 3028 & $\begin{array}{l}\text { Pneumokokken- } \\
\text { Gruppe }\end{array}$ \\
\hline $\begin{array}{l}39 \\
40\end{array}$ & $\begin{array}{l}\text { G. M. } \quad 2 \mathrm{~m} \\
\text { T. M. } \quad 47 \mathrm{~W}\end{array}$ & $\begin{array}{l}\text { Multiple subkntane } \\
\text { Abszesse }\end{array}$ & $\begin{array}{l}2585 \\
1465\end{array}$ & $\mathbf{x}$ \\
\hline & & Durehschnitt & $\begin{array}{c}0,0030823 \\
E(M)= \pm 0,00034654\end{array}$ & \\
\hline
\end{tabular}

werte des Lipasetiters waren bei pyogenem Eiter grösser als bei tuberkulösem. Hier muss jedoch berücksichtigt werden, dass sich bei meinen Fällen dem tuberkulösen Eiter aus den Nierenkavernen oft Harn beimengt fand und jener dadurch mehr oder weniger verdünnt war. Die relativ kleineren Werte des Lipasetiters bei unseren Fällen könnten davon herrühren. 
Tabelle IV. Lipase in tuberkulösem Eiter.

\begin{tabular}{|c|c|c|c|c|}
\hline $\begin{array}{l}\text { Fall- } \\
\mathrm{Nr} .\end{array}$ & $\begin{array}{l}\text { Name, Alter } \\
\text { a. Geschlecht }\end{array}$ & Kl. Diagnose & $\begin{array}{l}\text { Lipase (k) } \\
\text { im Eiter }\end{array}$ & $\begin{array}{c}\text { Tuberkelbazillen } \\
\text { im Eiter }\end{array}$ \\
\hline 41 & T. F. $19 \mathrm{~m}$ & $\begin{array}{l}\text { Senkungsabszess } \\
\text { bei Wirbeltbe. }\end{array}$ & 0,003451 & positiv \\
\hline 42 & K. S. $25 \mathrm{~m}$ & " & 4067 & $\mathbf{x}$ \\
\hline 43 & $\overline{\mathrm{Y}} . \mathrm{T} . \quad 25 \mathrm{~m}$ & Tbc. Empyem & 4067 & $\bar{x}$ \\
\hline 44 & $\mathrm{Y} . \mathrm{K} .16 \mathrm{w}$ & Kavern. Nierentbe. & 1369 & positiv \\
\hline 45 & J.I. $19 \mathrm{~m}$ & " & 2419 & " \\
\hline 46 & K.T. $19 \mathrm{w}$ & $"$ & 1879 & $"$ \\
\hline 47 & T. K. $21 \mathrm{w}$ & $"$ & 4067 & " \\
\hline 48 & T. K. $23 \mathrm{w}$ & " & 5177 & $"$ \\
\hline 49 & Y.S. $\quad 26 \mathrm{w}$ & " & 2739 & " \\
\hline 50 & K. U. $29 \mathrm{w}$ & $"$ & 3241 & $"$ \\
\hline 51 & S. Y. $30 \mathrm{~m}$ & ," & 0782 & ", \\
\hline 52 & T. T. $31 \mathrm{w}$ & $"$ & 3451 & $"$ \\
\hline 53 & K. S. 41 w & $"$ & 2048 & $"$ \\
\hline 54 & T. S. $48 \mathrm{w}$ & $"$ & 1051 & $"$ \\
\hline & & Durehschnitt & 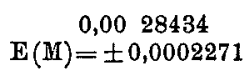 & \\
\hline
\end{tabular}

Nach Imok awas Untersuchungen wird die Lipasewirkung, im Gegensatz zur Annahme einiger früherer Autoren, dass sie für die Produkte bei chronischer Entzündung und besonders für den tuberkulösen Eiter spezifisch sei, weit stärker bei akut-entzündlichem Eiter gefunden. Takebaya shi wies aber in tuberkulösen Granulationsgeweben höheren Lipasegehalt nach. Ich habe bei pyogenem Eiter manchmal eine ebenso hohe oder sogar eine noch höhere Zahl des Lipasetiters als bei tuberkulösem Eiter festgestellt.

Bei Nierentuberkulose habe ich zuerst den Lipasetiter des Gesamtharns und des Blutserums, dann den der separierten Harne bestimmt, und zwar den jener zwei bei 16 Nierentuberkulosen (siehe Tabelle V), die verschiedene Stufen tuberkulöser Parenchymzerstörung aufwiesen. Hierbei war der Lipasetiter des Harns im allgemeinen deutlich hoch und zwar viel höher als bei Tuberkulösen ohne Nierenerkrankung und somit auch höher als bei Gesunden. Sein Durchschnittswert betrug 0,0010806. Auch der Lipasetiter des Blutserums verhielt sich hierbei ganz ähnlich wie der des Gesamtharns, sein Durchschnittswert betrug 0,0047308 .

Die Lipaseuntersuchung der separierten Harne stellte ich bei 11 Nierengesunden und 29 chirurgischen Nierenkranken an. Unter diesen befanden sich 14 Fälle von Nierentuberkulose, je ein Fall von Nierenkarbunkel und Nierensarkom, 3 Fälle von Nierensteinkrankheit, 4 von Wanderniere, ein Fall von Hydronephrose und 5 Fälle von Nephritis haematurica chronica. 
Tabelle V. Lipase in Gesamtharn und Blutserum bei Nierentuberkulose.

\begin{tabular}{|c|c|c|c|c|}
\hline \multirow{2}{*}{$\begin{array}{c}\text { Fall- } \\
\text { Nr. }\end{array}$} & \multirow{2}{*}{$\begin{array}{l}\text { Name, Alter } \\
\text { u. Geschlecht }\end{array}$} & \multirow{2}{*}{$\begin{array}{l}\text { K1. u. Operations- } \\
\text { Diagnose }\end{array}$} & \multicolumn{2}{|c|}{ Lipase $(k)$ in } \\
\hline & & & Harn & Blutserum \\
\hline $\begin{array}{l}55 \\
56 \\
57 \\
44 \\
45 \\
58 \\
48 \\
59 \\
49 \\
51 \\
52 \\
60 \\
54 \\
61 \\
62 \\
63\end{array}$ & 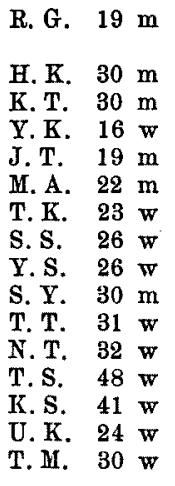 & 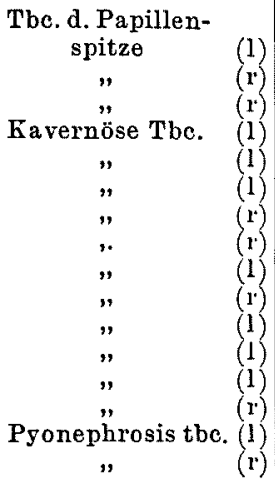 & $\begin{array}{rr}0,001 & 369 \\
& \\
\text { negativ } \\
0,000 & 782 \\
& 841 \\
& 841 \\
& 466 \\
2 & 163 \\
1 & 051 \\
& 719 \\
& 340 \\
& 782 \\
1 & 051 \\
& 782 \\
& 841 \\
& 500 \\
4 & 763\end{array}$ & $\begin{array}{r}0,004763 \\
5177 \\
5221 \\
4847 \\
4606 \\
5915 \\
6097 \\
3028 \\
5177 \\
4067 \\
4067 \\
5221 \\
4327 \\
3241 \\
5177 \\
4763\end{array}$ \\
\hline & & urohschnitt & $\begin{array}{c}0,0010806 \\
\mathrm{E}(\mathrm{M})= \pm 0,00017833\end{array}$ & $\begin{array}{c}0,0047308 \\
\mathrm{E}(\mathrm{M})= \pm 0,00013639\end{array}$ \\
\hline
\end{tabular}

Bei den Nierengesunden zeigte der Lipasetiter des separierten Harns auf beiden Seiten oft annähernd gleiche Wert oder Wert mit geringen Unterschieden (vgl. Fall 68, 69, 73 u. 74 in der Tabelle VI). Dagegen fand ich bei einigen Fällen (Fall 65, $70 \mathrm{u} .71$ ) auf einer Seite einen doppelt so grossen Lipasetiter wie auf der anderen Seite (vgl. Fall 71 in Tabelle VI).

Tabelle VI. Lipase in separierten Einzelnierenharnen.

\begin{tabular}{|c|c|c|c|c|c|c|}
\hline \multirow{2}{*}{$\begin{array}{c}\text { Fall- } \\
\text { Nr. }\end{array}$} & \multirow{2}{*}{$\begin{array}{l}\text { Name, Alter } \\
\text { u. Geschlecht }\end{array}$} & \multirow{2}{*}{ El. Diagnose } & \multicolumn{4}{|c|}{ Lipase (k) in separierten Halnen } \\
\hline & & & Rechts & (Blase) & Linl & ks \\
\hline 68 & $23 \mathrm{~m}$ & Nierengesunde & 0,000841 & $\mathrm{x}$ & 0,000 & 719 \\
\hline 69 & T. K. $23 \mathrm{w}$ & " & 340 & $0,000 \quad 474$ & & 474 \\
\hline 73 & I. S. $\quad 34 \mathrm{w}$ & $"$ & 782 & $\mathrm{x}$ & & 719 \\
\hline 74 & K. K. $\quad 37$ w & $"$ & 466 & $x$ & & 500 \\
\hline 71 & H. M. $28 \mathrm{~m}$ & $"$ & 340 & 474 & & 719 \\
\hline 76 & S. G. $23 \mathrm{~m}$ & Kavern. Nierentbc. (1) & 1274 & $\mathbf{x}$ & & 841 \\
\hline 82 & T. S. $48 \mathrm{w}$ & $"$ (1) & 719 & 340 & & $\mathrm{x}$ \\
\hline 78 & K. K. 26 w & (i) & 340 & 507 & & 507 \\
\hline 79 & T. T. $\quad 31 \mathrm{w}$ & (1) & 719 & 841 & & $\mathrm{x}$ \\
\hline 89 & Y.A. $28 \mathrm{w}$ & Nierenkarbunkel & 416 & $\mathbf{x}$ & & 340 \\
\hline 90 & T. $0.28 \mathrm{~m}$ & Nierensarkom & 841 & 614 & & 340 \\
\hline 93 & S. Y. $48 \mathrm{w}$ & Stein pyonephrosis (1) & 416 & 416 & 4 & 847 \\
\hline 94 & M. Y. $\quad 25 \mathrm{w}$ & Wanderniere & 782 & negativ & & 841 \\
\hline 103 & H. S. 51 w & Nephritis chr.haem. (1) & 719 & 500 & & 719 \\
\hline
\end{tabular}


Bei meinen Fällen von Nierentuberkulose, unter denen sich verschiedene Grade tuberkulöser Parenchymzerstörung und zwar Kavernenbildung feststellen liess, war der Lipasetiter oft (Fall 75, 76, 81, $82 \mathrm{u} .84$ ) auf der erkrankten Seite merklich vermindert (vgl. Fall 76 u. 82 in Tabelle VI). Bei den anderen Fällen (Fall 77-80, 83, 86 u. 88) wurde, im Gegensatz zu den obigen, der Lipasetiter auf der erkrankten Seite beträchtlich erhöht gefunden. Nur bei vereinzelten Fällen (Fall 85 u. 87) wies der Lipasetiter auf beiden Seiten gleiche Werte auf.

Bei Nierenkarbunkel und Nierensarkom (vgl. Fall 89 u. 90 in Tabelle VI) und einem Fall von Nierensteinkrankheit (Fall 91) verhielt sich der Lipasetiter auf der erkrankten Seite merklich vermindert. Bei einem anderen Fall von Nierensteinkrankheit, Steinpyonephrosis, (vgl. Fall 93 in Tabelle VI) war der Lipasetiter auf der erkrankten Seite stark erhöht, was meines Erachtens von der starken Beimengung mit Eiter herrühren dürfte. Bei Wandernieren wurde betreffs des Lipasetiters nur wenig Unterschied zwischen beiden Seiten bemerkt (siehe z. B. Fall 94 in Tabelle VI). Dieser Unterschied war bei Nephritis haematurica chronica nicht zu bemerken; die Lipasewerte blieben hierbei mitunter (siehe z. B. Fall 103 in Tabelle VI) trotz einseitiger starker Nierenblutung auf beiden Seiten beinah gleich.

Aus Obigem geht hervor, dass bei offener Nierentuberkulose weder der absolute noch auch der relative Wert des Lipasetiters des separierten Harns stets auf der tuberkulös erkrankten Seite erhöht gefunden wird. Der Lipasegehalt des tuberkulös erkrankten Nierenharns kann erstens von dem verschiedenen Beimengungsgrad tuberkulösen Eiters, also vom Grad der tuberkulösen Parenchymzerstörung, und zweitens von einer damit Hand in Hand gehenden Funktionsstörung der betreffenden Niere abhängen. Drittens muss man bei Bewertung des Lipasetiters des separierten Harns den Reiz des Ureterenkatheters, der oft Polyurie oder Oligurie der betreffenden oder der anderen Nierenseite auslöst, berücksichtigen. Das Auseinandergehen meiner Ergebnisse bei den Lipasetitern des separierten Harns könnte zum Teil durch solche Faktoren beeinflusst sein.

Auf Grund obiger Auseinandersetzungen kann ich wohl annehmen, dass trotz im allgemeinen erhöhtem Lipasegehalt des Gesamtharns und des Blutserums bei Nierentuberkulose seine Titerbestimmung in separierten Harnen sich zur spezifischen Diagnosestellung der Tuberkuloseerkrankung auf einer Nierenseite nicht anwenden lässt, und dass die vergleichende Untersuchung des Lipasegehalts in den separierten Nierenharnen erst dann Wert für die Funktionsprüfung der einzelnen Niere hat, wenn sie durch einige andere, bisher übliche $\mathrm{Me}$ thoden der Nierenfunktionsprüfung gestützt wird. Jedenfalls bleibt 
die genauere Lipaseforschung des separierten Harns bei Nierentuberkulose vorläufig noch weiteren Studien vorbehalten.

\section{Zusammenfassung.}

1. Ich stellte bei Nierentuberkulose zu chirurgisch-diagnostischem Zweck vergleichende Untersuchungen des separierten Harns auf Lipasegehalt an. Hierbei bestimmte ich zuerst den Lipasetiter des Gesamtharns und des Blutserums bei Gesunden und bei Tuberkulösen, unter Ausschluss von Nierenerkrankungen, dann aber auch bei Nierentuberkulose. Darauf habe ich bei Nierengesunden und chirurgischen Nierenerkrankungen, und zwar Nierentuberkulosen, den Lipasegehalt des separierten Harns untersucht. Bei allen diesen war eine gleichzeitige Erkrankung des Verdauungssystems, besonders des Pankreas, ganz ausgeschlossen. Ausserdem bestimmte ich auch den Lipasetiter des nichttuberkulösen und des tuberkulösen Eiters.

2. Der Lipasetiter des Harns und des Blutserums wurde nach der von Takebay as hi angegebenen Modifikation der Tributylinmethode nach Rona u. Micha elis bestimmt. Hierbei wurde der Harn in nativem Zustande, das Blutserum in 10 facher und der Eiter in 5facher Verdünnung, von allen je $2 \mathrm{ccm}$, nach der Originalmethode verwendet.

3. Bei Gesunden betrug der Durchschnittswert des Lipasetiters des Harns 0,0006893 und der des Blutserums 0,0038612. Die Schwankungen zwischen den einzelnen Wert waren beim Harn über 2 fach so gross wie beim Blutserum.

4. Bei Kranken mit chirurgischer oder innerer Tuberkulose ohne Nierenerkrankungen wurde der Durchschnittswert des Lipasetiters des Harns und des Blutserums weit höher gefunden als bei Gesunden. Hier verhielten sich die Schwankungen der einzelnen Lipasewerte des Harns und des Blutserums beinah wie bei Gesunden.

5. Der Lipasetiter des tuberkulösen Eiters und auch des Inhalts derNierenkaverne wal nicht immer grösser als der des pyogenen Eiters.

6. Der Lipasetiters des Gesamtharns war bei Nierentuberkulose im allgemeinen hoch, und zwar viel höher als bei Tuberkulösen ohne Nierenerkrankungen, somit auch viel höher als bei Gesunden. Ebenso verhielt sich der Lipasetiter des Blutserums.

7. Der Lipasetiter des separierten Einzelnierenharns wurde bei Nierengesunden auf beiden Seiten, mit wenigen Ausnahmen, annähernd gleich oder nur wenig verschieden gefunden. Bei einseitiger Nierentuberkulose, und zwar bei kavernöser, war der Lipasetiter auf der erkrankten Seite oft deutlich vermindert, ebenso häufig aber merklich erhöht, so d'ass sich daraus nichts Bestimnites ergab. Bei einem Fall von Steinpyoneplrosis wurde der Lipasetiter auf der erkrankten Seite 
enorm erhöht gefunden, was sich wohl auf starke Eiterbeimengung im betreffenden Nierenharn zurückführen lässt.

8. Weder der absolute noch auch der relative Wert des Lipasetiters des separierten Harns wurde bei Nierentuberkulose auf der tuberkulös erkrankten Seite stets erhöht gefunden, oft sogar im Gegenteil vermindert. Daraus darf ich wohl schliessen, dass die Lipasebestimmung des separierten Harns bei Nierentuberkulose nicht für die spezifische Diagnose der tuberkulösen Erkrankung in einer Niere, sondern höchstens für die Funktionsprüfung der einzelnen Niere, und zwar erst unter Kontrolle der anderen bisherigen Methoden der Nierenfunktionsprüung, dienen kann. Deshalb sind noch weitere Studien hierüber sehr erwünscht.

\section{Literatur.}

(1) Arndt, K.; Stalagmometer von J. Traube. Handbuch der physikalisehchemischen Technik. 2. Aufl. Stuttgart 1923, 589-590.

(2) Ba ch, E.; Ztschr. kl. Med., 1922, 95, 103-108.

(3) Bantin, E.; Ref. : Kongressztrlbl. ges. inn. Med., 1927, 46, 255.

(4) Bauer, J.; Wien. kl. Wochenschr., 1912, 1376-1380.

(5) Berg, S. ; Acta med. Scandinav., 1929, 72, 309-325.

(6) Brugseh, T. u. A. Sehittenhelm; Klinische Laboratoriumtechnik. 2. Aufl.

Bd. II. Berlin u. Wien 1924, 1066-1069 u. 1405-1418.

(7) Ca mero n, G.; Med. Journ. Australia, 1923, 408-411.

(8) Fiessinger, N.; Les ferments des leucocytes. Paris 1923, 149-185.

(9) Frisch, A. u. V. Kollert; Beitr. Kl. d. Tuberkul. 1921, 47, 146-159.

(10) H a h, F.-V. v.; Münch. med. Wochenschr., 1929, 1964-1965.

(11) Imok aw a, T.; Kaigun Gunikai Zasshi, 1927, 16, 111-138.

(12) I $\mathrm{l}_{\mathrm{g}} \mathrm{er}$, J. u. J. S a piro ; Ref. : Ztrlorg. ges. Chir., 1930, 51, 194-195.

(13) Johannsen, W.; Elemente der exakten Erblichkeitslehre. 3. Aufl. Jena 1926,43 u. 97

(14) Kollert, V. u. A. Friseh ; Beitr. Kl. d. Tuberkul., 1920, 43, 305-326.

(15) K a ramochi, K.; I,ji Shimbun, 1930, Nr. 1258, 61-93.

(16) Molcanova, V. u. M. Nizuvska,ja; Ztrlorg. ges. Chir., 1930, 50, 133-134:

(17) Nakajima, K. u. K. Mizuno; Hokkaido Igakkai Zasshi, 1924, 1, 263-282.

(18) Nicolau, I. u. O.Antinescu; Ref.: Kongressztrlbl.ges. inn. Med., 1929, 52, 442.

(19) Oppenheimer, C.; Die Fermente und ihre Wirkungen. 4. Aufl. Bd. I. Leipzig 1913, 157-178.

(20) Res c h, A.; Dtsch. Arch. kl. Med., 1916, 118, 179-189.

(21) Ron a, P. u. L. Hichaelis; Biochem. Ztschr., 1911, 31, 345-354.

(22) Rosows ky, F.; Med. Klin., 1926, 178-179.

(23) Ruszt, I. ; Ref: Ztschr. urol. Chir., 1927, 23, 39.

(24) S a g a l, Z.; Journ. med. Research, 1916, 34, 231-240.

(25) Schranz, H.; Arch. kl. Chir., 1925, 134, 200-202.

(26) Sorkin, A.; Ref: Ztrlorg. ges. Chir., 1929, 46, 15.

(27) Sue ok a, S.; Okayama Igakkai Zasshi, 1930, 42, 370-387.

(28) T a ke ba y a shi, H. ; Beitr. Kl. d. Tuberkul., 1927, 67, 748-755.

(29) Ts u.ji, Y.; Chugai Iji Shimpo, 1924, Nr. 1059, 645-662.

(30) Wohlgemuth, J.; Grundriss der Fermentmethoden. Berlin 1913, 101-134.

(31) Ya ma moto, H. ; Tohoku Journ. Exp. Med., 1929, 13, 568-579. 